\title{
Imipenem-resistant Pseudomonas aeruginosa treated with piperacillin/tazobactam in a patient with severe pneumonia: a case report and related literature review
}

\author{
Lu He ${ }^{1}$, Aiguo $\mathrm{He}^{2}$, Yan Qian ${ }^{1}$ \\ ${ }^{1}$ Department of Pharmacy, The Second Affiliated Hospital of Chongqing Medical University, Chongqing, China; ${ }^{2}$ Department of Oncology, Zigong \\ Second People's Hospital, Zigong, China \\ Correspondence to: Yan Qian. Department of Pharmacy, The Second Affiliated Hospital of Chongqing Medical University, Chongqing, China. \\ Email: cqqianyan@hospital.cqmu.edu.cn.
}

\begin{abstract}
Pseudomonas aeruginosa (PA) is a common gram-negative bacterium. Imipenem (IMP) is considered to be the most effective clinical drug for the treatment of PA infection. IMP-resistant ceftazidimesusceptible PA is relatively rare in clinical practice; so far, there have been no clinical reports regarding the treatment of IMP-resistant PA with piperacillin/tazobactam alone. This paper will report the case of a severe pneumonia patient with IMP-resistant ceftazidime-susceptible PA infection that was successfully treated with piperacillin/tazobactam monotherapy after initial therapy with biapenem (BIP) according to the drug sensitivity test. The patient was a 75-year-old female, her main symptom was drowsiness. She was admitted to hospital due to acute exacerbation of chronic obstructive pulmonary disease (AECOPD) and pulmonary encephalopathy. After admission, endotracheal intubation was performed immediately, and the lavage fluid was sent to the laboratory for sputum culturing for several times. BIP was selected for the initial anti-infection regimen, and other symptomatic treatments were performed at the same time. On the day 8 , the sputum culture drug sensitivity test results showed PA (sensitive to: piperacillin/tazobactam sodium, ceftazidime, aminoglycoside, and fluoroquinolones; resistant to IMP; and intermediate sensitivity to: cefoperazonesulbactam and meropenem), so we adjusted the anti-infection regimen as piperacillin/tazobactam sodium. After that, the infection index of the patient declined steadily, and the patient was discharged from hospital after continuous treatment with piperacillin tazobactam until the 24th day. For severe pneumonia patients with IMP-resistant ceftazidime-susceptible PA infection, piperacillin/tazobactam is still an option specially when the MIC of piperacillin/tazobactam is very low.
\end{abstract}

Keywords: Pseudomonas aeruginosa (PA); carbapenem resistance; ceftazidime susceptible; piperacillin/ tazobactam; case report

Submitted Nov 21, 2020. Accepted for publication Jan 17, 2021.

doi: 10.21037/apm-20-2495

View this article at: http://dx.doi.org/10.21037/apm-20-2495

\section{Introduction}

Pseudomonas aeruginosa (PA) is a common gram-negative bacterium. It does not usually show pathogenicity in healthy populations, but can easily induce infection and is difficult to cure in patients with low resistance and accompanying partial pulmonary organic lesions (1). According to CHINET data in 2019, PA was ranked third among the strains isolated from respiratory tract specimens and is one of the main pathogens causing nosocomial infections.

Carbapenems belong to a class of antibacterial agents known as non-typical $\beta$-lactamides, and include drugs such as imipenem (IMP), meropenem (MEM), and biapenem (BIP). The World Health Organization (WHO) has recently reclassified carbapene-resistant $\mathrm{PA}$ as one of the three bacteria in urgent need of new antibiotics (1). IMP has been considered to be the most effective clinical drug for the treatment of 
PA infection. It is a broad-spectrum antibiotic with strong antibacterial activity, and acts by inhibiting the synthesis of the bacterial cell wall. In recent years, The Chinese CHINET system reported that the drug resistance rate of PA to IMP has been maintained at about 30\% in China. Since IMP-resistant $\mathrm{PA}$ infection is often multidrug-resistant, such infections can triple the increase in the mortality rate, increase the rate of secondary bacteremia by nine times, and double the average length of hospital stay compared with ordinary infections (2). IMP-resistant ceftazidime-susceptible PA is relatively rare in clinical practice; so far there have been no clinical reports regarding the treatment of IMP-resistant PA with piperacillin/ tazobactam alone. One study showed that the therapeutic effect of other $\beta$-lactam drugs in IMP-resistant ceftazidimesusceptible PA infection would be inadequate (3). In this case, we used BIP as an initial therapy, and subsequently adjusted to piperacillin/tazobactam according to the drug-sensitivity test. We successfully cured an IMP-resistant ceftazidimesusceptible PA infection in a patient with severe pneumonia. We present the following article in accordance with the CARE reporting checklist (available at http://dx.doi.org/10.21037/ apm-20-2495).

\section{Case presentation}

\section{Basic information}

A 75-year-old female patient was admitted to hospital due to repeated cough and expectoration of sputum for more than 20 years, aggravation for 5 days, and drowsiness for 1 day. In the past, the patient may have coughed and expectorated after a cold, with mostly white bubbly sputum. There was no discomfort such as dyspnea or hemoptysis. The patient had been self-administering salmeterol ticasone inhalant 250/50 $\mu \mathrm{g}$ and tiotropium bromide spray long-term, however, symptoms returning after a cold would often require hospitalization. The patient was diagnosed with chronic obstructive pulmonary disease (COPD) in another hospital. After each attack, she could be cured with anti-infection (details are unknown), bronchiectasis, cough, and expectorant symptomatic treatment. On June 17, 2020, the patient was hospitalized due to the aggravation of the aforementioned symptoms. Chest CT (on June 19) showed varicose dilatation with infection in part of the bronchi of both lungs. On June 22, the patient developed drowsiness, no fever, unconscious loss, and no obvious cough or expectoration. On June 23, the patient presented with incoherent answers, was unable to recognize people, and later presented with consciousness disorder. An arterial blood gas
(ABG) [fraction of inspired oxygen $\left(\mathrm{FiO}_{2}\right) 33 \%$ ] test revealed the following: $\mathrm{pH} 7.39$, partial pressure of oxygen $\left(\mathrm{PO}_{2}\right)$ $108 \mathrm{mmHg}$, partial pressure of carbon dioxide $\left(\mathrm{PCO}_{2}\right)$ $125 \mathrm{mmHg}$, bicarbonate $\left(\mathrm{HCO}_{3-}{ }^{-}\right) 73.2 \mathrm{mmol} / \mathrm{L}$. The patient was then transferred to our hospital as her condition worsened. She had a 2-year history of hypertension and denied any history of food or drug allergy and family. Written informed consent was obtained from the patient for publication of this study and any accompanying images. All procedures performed in studies involving human participants were in accordance with the ethical standards of the institutional and/or national research committee(s) and with the Helsinki Declaration (as revised in 2013). Written informed consent was obtained from the patient.

\section{Hospitalization condition}

The patient's physical examination upon hospitalization was as follows: temperature, $36.7^{\circ} \mathrm{C}$; heart rate (HR), $112 \mathrm{bpm}$; respiration, $19 \mathrm{bpm}$; blood pressure $(\mathrm{BP}), 148 / 97 \mathrm{mmHg}$. She was suffering from a blurred mind and was sleepy, as well as coarse breathing and a wheeze in both lungs. ABG ( $\mathrm{FiO}_{2} 37 \%$ ) showed the following: $\mathrm{pH}, 7.41, \mathrm{PO}_{2}, 56 \mathrm{mmHg}$, $\mathrm{PCO}_{2}, 126 \mathrm{mmHg}, \mathrm{HCO}_{3},-58.2 \mathrm{mmol} / \mathrm{L}$, and oxygenation index, 151. Laboratory tests revealed the following: white blood cell (WBC) count, $10.33 \times 10^{9} / \mathrm{L}$; neutrophils percentage (NEUT\%), 76.9\%; C-reactive protein (CRP), $<5.0 \mathrm{mg} / \mathrm{L}$. Calcitonin was $0.085 \mathrm{ng} / \mathrm{mL}$, serum potassium was $2.24 \mathrm{mmol} / \mathrm{L}$, serum chlorine was $71 \mathrm{mmol} / \mathrm{L}$, serum magnesium was $0.35 \mathrm{mmol} / \mathrm{L}$, endogenous creatinine clearance rate was $62.2 \mathrm{~mL} / \mathrm{min}$, and the glomerular filtration rate was $59.8 \mathrm{~mL} / \mathrm{min}$. A chest X-ray showed inflammation in the patient's lungs (Figure 1). The patient was diagnosed with the following: (I) pulmonary encephalopathy; (II) acute exacerbation of COPD (AECOPD); (III) type II respiratory failure; (IV) bronchiectasis accompanied by infection; (V) chronic pulmonary heart disease; (VI) hypertension.

\section{Procedures}

The patient was admitted to the intensive care unit (ICU) according to the APACHE II (Acute Physiology and Chronic Health Evaluation II) [34] and Sepsis related organ failure assessment (SOFA) [13] scores. After admission, her HR gradually decreased to $43 \mathrm{bpm}$. Endotracheal intubation was immediately performed and was connected to a ventilator for mechanical ventilation, and dopamine was pumped in at $6 \mu \mathrm{g} / \mathrm{min}$. The patient then underwent 


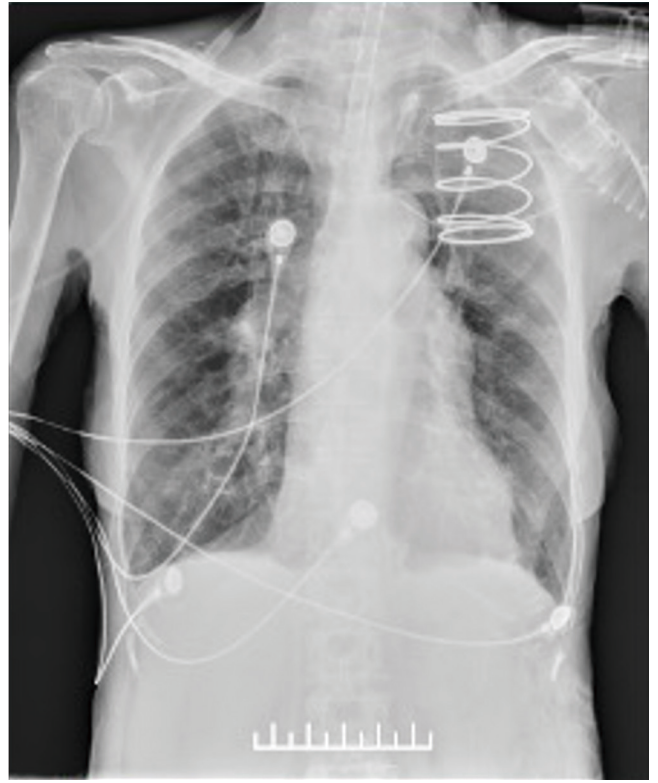

Figure 1 2020.06.23 X-ray chest radiograph.

bronchoalveolar lavage, and the lavage fluid was sent to the laboratory for sputum culturing. At the same time, BIP $0.3 \mathrm{~g}$, ivgtt, $\mathrm{q} 8 \mathrm{~h}$, was used as an anti-infective treatment, budesonide combined with salbutamol (atomized inhalation) was used to treat bronchiectasis, acetylcysteine (atomized inhalation) was used for sputum, arginine (injection) was administered to correct alkalosis, and potassium magnesium aspartate and potassium chloride were given to correct the patient's electrolyte imbalance. After symptomatic treatment, $\mathrm{ABG}\left(\mathrm{FIO}_{2} 40 \%\right)$ showed the following: $\mathrm{pH}, 7.65 ; \mathrm{PO}_{2}, 89 \mathrm{mmHg} ; \mathrm{PCO}_{2}, 58 \mathrm{mmHg} ; \mathrm{HCO}_{3}^{-}$, $53.4 \mathrm{mmol} / \mathrm{L}$; and oxygenation index, 223.

On day 3 , the patient was still unconscious without response, however her breathing was stable. ABG $\left(\mathrm{FIO}_{2}\right.$ $40 \%$ ) revealed the following: $\mathrm{pH}, 7.54 ; \mathrm{PO}_{2}, 149 \mathrm{mmHg}$; $\mathrm{PCO}_{2}, 38 \mathrm{mmHg} ; \mathrm{HCO}_{3}-, 32.2 \mathrm{mmol} / \mathrm{L}$; and oxygenation index, 373. Her laboratory test showed the following: WBC count, $12.48 \times 10^{9} / \mathrm{L}$; NEUT\%, $81.3 \%$; and CRP, $58.63 \mathrm{mg} / \mathrm{L}$. Lactic acid was $4.7 \mathrm{mmol} / \mathrm{L}$, and the sputum culture was negative. Although the infection index of the patient increased, the ABG suggested that her pulmonary function was improved. Considering the effectiveness of the patient's treatment regimen, the sputum culture was continued without adjustment of antibacterial therapy.

On day 6, the patient was still delirious and unresponsive, and was breathing steadily. ABG $\left(\mathrm{FIO}_{2}\right.$ $35 \%$ ) showed the following: $\mathrm{pH}, 7.5 ; \mathrm{PO}_{2}, 106 \mathrm{mmHg}$;
$\mathrm{PCO}_{2}, 37 \mathrm{mmHg} ; \mathrm{HCO}_{3}-, 32.8 \mathrm{mmol} / \mathrm{L}$; and oxygenation index, 297. Laboratory test results were as follows: WBC count, $9.42 \times 10^{9} / \mathrm{L}$; NEUT\%, $79.2 \%$; and CRP, $93.31 \mathrm{mg} / \mathrm{L}$. Repeat chest X-ray suggested that the patient's pulmonary inflammation was decreased. The sputum culture was negative. The patient's condition at this stage was stable, and the chest $\mathrm{X}$-ray indicated an improvement in pulmonary inflammation. Considering the effectiveness of the treatment regimen, the patient continued with antiinfection therapy.

On day 8 , the patient remained delirious; she was breathing steadily and was unresponsive. $\mathrm{ABG}\left(\mathrm{FIO}_{2} 30 \%\right)$ showed the following: $\mathrm{pH}, 7.48 ; \mathrm{PO}_{2}, 88 \mathrm{mmHg} ; \mathrm{PCO}_{2}$, $41 \mathrm{mmHg} ; \mathrm{HCO}_{3}-, 29.9 \mathrm{mmol} / \mathrm{L}$; and oxygenation index, 293. Laboratory tests revealed the following: WBC count, 9.56 $\times 10^{9} / \mathrm{L}$; NEUT\%, 73.1\%; and CRP $74.09 \mathrm{mg} / \mathrm{L}$. The sputum culture drug sensitivity test results showed PA (sensitive to: piperacillin/tazobactam sodium, ceftazidime, aminoglycoside, and fluoroquinolones; resistant to IMP; and intermediate sensitivity to: cefoperazone-sulbactam and MEM; Table 1). The antibacterial drug was adjusted to piperacillin/tazobactam sodium $4.5 \mathrm{~g}$, IVGTT, q8h according to the patient's drug sensitivity test.

On day 11, the patient was conscious and had no complaints of special discomfort. Her laboratory tests showed the following: WBC count, $9.15 \times 10^{9} / \mathrm{L}$; NEUT\%, $74.9 \%$; and CRP $44.07 \mathrm{mg} / \mathrm{L}$. After assessment, mechanical ventilation was suspended at 17:30, the endotracheal tube was pulled out, and a non-invasive ventilator that assists ventilation was applied to the patient's facemask. ABG $\left(\mathrm{FIO}_{2} 35 \%\right)$ revealed the following: $\mathrm{pH}, 7.42 ; \mathrm{PO}_{2}, 101 \mathrm{mmHg} ; \mathrm{PCO}_{2}, 47 \mathrm{mmHg}$; $\mathrm{HCO}_{3}-, 29.2 \mathrm{mmol} / \mathrm{L}$; and oxygenation index, 289.

Following this, the patient's infection index declined steadily, the blood gas index was stable, and the anti-infection therapy was continued. On day 20, we reviewed the patient's chest $\mathrm{X}$-rays. The Chest $\mathrm{X}$-rays showed significant relief of lung inflammation for the patient (Figure 2). On day 24, the patient's symptoms improved and she was discharged from the hospital. We assessed that the above treatment regimen was effective and no adverse drug reactions occurred during the treatment,and the patient did not complain of special discomfort.

\section{Discussion}

\section{Mechanism of resistance of PA to carbapenems}

Recently, with the extensive use of broad-spectrum 


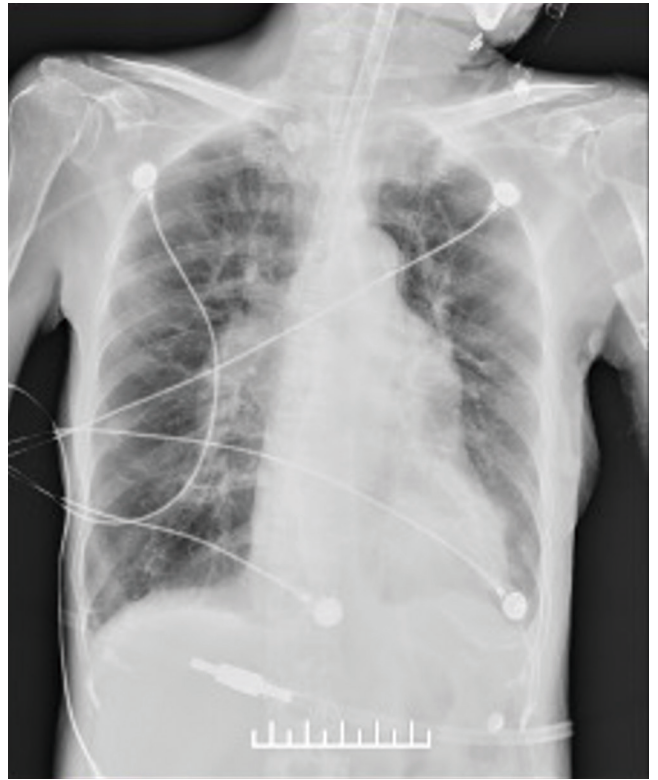

Figure 2 2020.07.03 X-ray chest radiograph.

antibacterial drugs, PA resistance is becoming increasingly serious. Previous studies have shown that the drug resistance mechanisms of PA primarily include antibioticinactivating enzymes, changes in drug targets and outer membrane permeability, active efflux systems, and the formation of bacterial biofilm. The mechanism leading to PA resistance to carbapenems mainly involves the following aspects:

\section{Mutation or deletion of the outer membrane protein OprD2}

Antibiotics targets are located in the cell or intracellular membranes of bacteria, and the drug must enter the bacterial body through the outer membrane before contacting the target. The epicyte of PA has microporous protein channels formed by various outer membrane proteins, and only some antibacterial drugs are able to pass through. The outer membrane protein, $\mathrm{OprD}$, is a specific channel for IMP. It is generally believed that mutation or deletion of the OprD protein gene leads to decreased OprD protein, which is the main mechanism of $\mathrm{PA}$ resistance to carbapenems (especially IMP). The structure of the OprD protein consists of eight rings, of which two rings (amino acid site 96-134) contain IMP-specific binding sites, and three rings (amino acid site 157-203) are the entry channels of IMP into the bacteria (4), with no cross-resistance to other $\beta$-lactam antibiotics (including MEM) $(5,6)$.

\section{Efflux systems}

Efflux systems are a group of outer membrane proteins in PA cells that are closely related to bacterial drug resistance. The RND family efflux pumps are related to PA drug resistance. There are 12 kinds of RND efflux pumps in total, of which MexAB-OprM, MexCDOprJ, MexEF-OprN, and MexXY-OprM are correlated with antimicrobial resistance. The MEXAB-OprM and MexCD-OprJ efflux pumps can act on $\beta$-lactams (1), and MexAB-OprM is the most important drug resistance mechanism. The overexpression of MexAB-OprM is the key factor leading to MEM resistance in PA, and can even lead to ceftazidime/avibatan resistance, however has little effect on IMP (7-9).

\section{Production of lactamase}

$\beta$-lactamases can be divided into four classes, A, B, C, and $\mathrm{D}$, based on their amino acid sequences. $\mathrm{A}, \mathrm{C}$, and $\mathrm{D}$ are lactamases, while $B$ is metal $\beta$-lactamase (MBL). The $\beta$-lactamase produced by PA mainly includes extendedspectrum beta-lactamases (ESBLs), the AmpC enzyme, and MBL. MBL is characterized by resistance to most $\beta$-lactamases, carbapenems, and enzyme inhibitors, resulting in pan-resistance to PA. So far, IMP, VIM, SPM, GIM, and AIM type MBLs have been detected in PA, with IMP and VIM types being more common than other types. MBL is one of the main factors that leads to carbapenem resistance $(10,11)$. AmpC enzymes are class $C$ enzymes, and are characterized by resistance to cephalosporin and cephalomycin, as well as insensitivity to enzyme inhibitors. Studies have shown that AmpC enzymes are also resistant to carbapenems to a certain extent (12). Under normal circumstances, only a small amount of $\mathrm{AmpC}$ is produced when there is no inducer effect. However, an inducer effect such as the use of a large amount of third-generation cephalosporin can greatly increase its expression level, indicating that the unreasonable use of antibiotics in clinical practice will result in the widespread prevalence of the AmpC enzyme PA strain. ESBLs are class A enzymes, and are characterized by their ability to hydrolyze broadspectrum penicillin, third-generation cephalosporins, and monocyclic antibiotics. However, they are sensitive to cephalosporins, carbapenems, and enzyme inhibitors (13). 
Table 1 Sputum culture drug sensitivity test results

\begin{tabular}{lcc}
\hline Drug & MIC & Result \\
\hline Gentamicin & $\leq 1$ & $\mathrm{~S}$ \\
Amikacin & $\leq 2$ & $\mathrm{~S}$ \\
Isepamicin & $\leq 4$ & $\mathrm{~S}$ \\
Piperacillin tazobactam & 16 & $\mathrm{~S}$ \\
Cefoperazone sulbactam & 4 & $\mathrm{I}$ \\
Doripenem & $\geq 16$ & $\mathrm{I}$ \\
Imipenem & 4 & $\mathrm{R}$ \\
Meropenem & $\leq 8$ & $\mathrm{I}$ \\
Ticarcillin & $\leq 4$ & $\mathrm{~S}$ \\
Piperacillin & $\leq 1$ & $\mathrm{~S}$ \\
Aztreonam & 2 & $\mathrm{~S}$ \\
Ceftazidime & $\leq 1$ & $\mathrm{~S}$ \\
Cefepime & $\leq 0.25$ & $\mathrm{~S}$ \\
Ciprofloxacin & $\leq 0.12$ & $\mathrm{~S}$ \\
Levofloxacin & $\mathrm{S}$ \\
\hline M C, minimum & & $\mathrm{s}$ \\
\hline
\end{tabular}

MIC, minimum Inhibitory Concentration; S, sensitive; I, intermediate; $\mathrm{R}$, resistant.

\section{Penicillin binding proteins (PBPs)}

$\mathrm{PBP}$ is a special protein molecule that exists in the membrane of the bacterial cytoplasm, and can contribute to the biosynthesis of peptidoglycan in the bacterial cell wall. $\beta$-lactam antibiotics can bind to it to inhibit the formation of the cell wall and play a bactericidal role. PA has eight PBPs; PBP2 and PBP3 are necessary to maintain normal form and bacterial survival, and IMP is highly dependent on PBP2. So, it is likely that PBP2 is also the target of IMP (14). However, there has been no definitive study demonstrating the relationship between $\mathrm{PBP} 2 / \mathrm{PBP} 3$ and carbapenem antibiotic resistance.

\section{Biofilm formation}

Bacteria can secrete a polysaccharide matrix and fibrin upon contact with the surface of inert objects to escape the killing effect of immunity and antibacterial drugs. This is an adaptive mechanism for bacteria in their environment; with strong drug resistance, bacteria can resist more than 100 times the normal drug dose (1). Alginate polysaccharide is the main component of the PA biofilm matrix, which can hinder the penetration of antibacterial drugs and can also combine with a variety of hydrolases to inactivate antibacterial drugs before they enter the cells. With the wide application of medical materials and the increasing utilization of various catheterization operations, PA can easily form biofilm, which leads to infection. The formation of biofilm has nothing to do with drug induction, and the specificity of the drug resistance mechanism is not significant.

Wi et al. (3) screened three IMP-resistant ceftazidimesusceptible PA isolates, and ceftazidime, cefepime, and piperacillin/tazobactam were used for sensitivity tests in vitro. Their results suggested that although the three $\beta$-lactam antibiotics showed significant killing effects with the standard inocula, they had no effect with the high inocula. Therefore, $\beta$-lactam monotherapy may not be effective against IMP-resistant PA infections with large bacterial burdens, despite their susceptibility to $\beta$-lactams. However, the minimum inhibitory concentration (MIC) values of the three $\mathrm{PA}$ strains selected in this study were $16 / 4,8 / 4$, and $1 / 4$, respectively, and the MIC value of PA with $1 / 4$ was significant in both high and low inocula bacteria.

The patient's sputum culture result indicated growth of PA (Table 1). The results showed that only IMP was resistant, MEM $(\mathrm{MIC}=4)$ and cefoperazone-sulbactam were intermediate, and other $\beta$-lactams, amino glycosides, and levofloxacins were sensitive. It can be inferred that the key mechanism of PA infection in this patient may have been a decrease in the expression of the OprD2 membrane porin, accompanied by a possible combination of increased MexAB-OprM efflux system expression and changes in the PBP2 target, rather than the usual MBL enzymes. At the same time, the above mechanism has high specificity and the MIC value of piperacillin/tazobactam is low, and thus, we believe that it was feasible to use piperacillin/tazobactam in this case.

\section{Was the patient's anti-infective therapy reasonable?}

Patients that meet one of the following major criteria or at least three of the following minor criteria can be diagnosed with severe pneumonia. The major criteria are as follows: (I) mechanical ventilation is required for tracheal intubation; and (II) vasoactive drugs are still required after active fluid resuscitation in septic shock. The minor/secondary criteria include the following: (I) respiratory rate $>30 \mathrm{bpm}$; (II) $\mathrm{PaO}_{2} / \mathrm{FiO}_{2}<250 \mathrm{mmHg}$; (III) multilobar infiltration; 
(IV) disturbance of consciousness and/or disorientation; (V) blood urea nitrogen $\geq 7 \mathrm{mmol} / \mathrm{L}$; and (VI) low blood pressure requiring active fluids (15). Also, patients with AECOPD combined with increased dyspnea, increased sputum volume, and two basic symptoms of purulent sputum (including increased purulent sputum), or those needing invasive or non-invasive mechanical ventilation treatment should receive antibiotic treatment (16).

AECOPD patients with any of the following two adjustments may be considered to exhibit high risk factors for PA infection: (I) hospitalization history of approximately 1 year; (II) frequent ( $>4$ times/year) or recent (3 months) history of antibacterial drug use; (III) extremely severe COPD (FEV1 (Forced expiratory volume in one second) $\%<30 \%$ ); (IV) those taking prednisone $>10 \mathrm{mg} / \mathrm{d}$ in the past 2 weeks; and (V) previous history of PA infection (17). Risk factors for multi-drug resistant bacterial infection include: (I) old age; (II) low immune function; (III) central venous intubation, mechanical ventilation, urinary tract intubation, and other invasive procedures; (IV) patients that recently (within 90 days) received more than three kinds of antibacterial drugs; (V) multiple or long-term hospitalization history; and (VI) a history of colonization or infection with multidrug-resistant bacteria (18).

The patient in our study had progressed to type II respiratory failure and was given invasive respiratory ventilation upon admission. Her $\mathrm{PaO}_{2} / \mathrm{FiO}_{2}$ was $<250 \mathrm{mmHg}$ and she had accompanying consciousness disorder, which met the diagnostic criteria for severe pneumonia. Anti-infection treatment should be given immediately in such cases. Furthermore, the patient is elderly (75 years old) with a long-term history of COPD and at least one hospitalization in the past year (the hospitalization history of this patient in our hospital in the past year can be found). Before admission, the patient was given antibiotics in another hospital, but the treatment effect was ineffective. After admission, the patient received mechanical ventilation. These factors support the necessary of the patient to consider the high risk of multidrug-resistant PA infection. According to the current guidelines, for AECOPD patients with severe pneumonia who are at high risk of multidrug-resistant PA infection, the recommended initial regimen is combination therapy in order to cover possible pathogenic bacteria, and is more effective than single-drug therapy (19).

The patient was initially treated with BIP $0.3 \mathrm{~g}$, IVGTT, Q8h for 8 days in our hospital. After positive sputum culture results were obtained on the 8 th day and the patient's situation was assessed, she was adjusted to piperacillin/ tazobactam $4.5 \mathrm{~g}$, IVGTT, Q8h according to the drug sensitivity test. Our prior analysis showed that the patient exhibited risk factors multiple-drug resistant PA infection, and in this case, $\beta$-lactams with enzyme inhibitors combined with aminoglycosides or fluoroquinolones with anti-PA activity was the preferred therapy option. However, the patient only used BIP, and the risk of failure was substantial. On the other hand, the aforementioned combination regimen can reduce the exposure to carbapenem, thus reducing the resistance to carbapenem antibiotics. Thus, we believe that the combination regimen is more appropriate.

The patient received antimicrobial therapy for a total of 24 days. For patients with severe AECOPD combined with bronchiectasis and mechanical ventilation, as well as clear PA infection, the recommended course of treatment is 10-14 days. There are also guidelines that recommend antibacterial therapy for $>2$ weeks for severe pneumonia patients infected with $\mathrm{PA}$, which generally extend to $3-5$ days after heat regression or significant improvement of major respiratory symptoms. In this case, the electrolyte imbalance (blood potassium, blood chlorine, and blood magnesium) of the patient returned to normal on the 9th day. Her acid-base imbalance was completely corrected on the 10th day, $\mathrm{PO}_{2}$ and $\mathrm{PCO}_{2}$ reached normal levels, $\mathrm{SO}_{2}$ was more than $90 \%$, the oxygenation index was 300 , and no recurrence occurred. On the 11th day of treatment, mechanical ventilation was suspended, and mask-assisted ventilation was administered. The patient's consciousness was restored, her breathing was stable, and no rashes or wheezing were observed in both lower lungs. On the 14th day, WBC count and NEUT\% returned to normal.

Assessing the patient's condition, it was appropriate for her to cease antibiotic treatment 3-5 days after the infection index returned to normal. If so, the antimicrobial course would have been 17-19 days, which has reached the course of severe pneumonia caused by PA infection. However, the patient received anti-infection treatment for a total of 24 days; this treatment course was excessively long, which may lead to drugresistant bacteria.

\section{Conclusions}

The treatment of the patient in this case was generally successful. From this case, we can see that for the patients who are infected with the IMP-resistant ceftazidimesusceptible PA, piperacillin/tazobactam may still be effective if the drug sensitivity test is performed and the MIC value of piperacillin/tazobactam is very low. However, these 
conclusions still need to be verified in broader clinical trials. At the same time, there were still some disadvantages during the anti-infection treatment of this patient, such as the high-risk drug selection in the initial treatment regimen and the long duration of antimicrobial therapy. Excessive use of carbapenems and prolonged use of antibiotics will greatly increase the possibility of drug-resistant bacteria, and may ultimately lead to an absence of available drug options for these patients in the future. Also, for patients at risk of infection with multi-drug resistant bacteria, whether carbapenem monotherapy or in combination with lowerlevel antibiotics is a better choice as the initial treatment regimen requires further study in the future.

\section{Acknowledgments}

Funding: Social Undertakings and Livelihood Security Science and Technology Innovation Project of Chongqing Science and Technology Commission (cstc2017shmsA130041).

\section{Footnote}

Reporting Checklist: The authors have completed the CARE reporting checklist. Available at http://dx.doi.org/10.21037/ apm-20-2495

Conflicts of Interest: All authors have completed the ICMJE uniform disclosure form (available at http://dx.doi. org/10.21037/apm-20-2495). The authors have no conflicts of interest to declare.

Ethical Statement: The authors are accountable for all aspects of the work in ensuring that questions related to the accuracy or integrity of any part of the work are appropriately investigated and resolved. Written informed consent was obtained from the patient for publication of this study and any accompanying images. All procedures performed in studies involving human participants were in accordance with the ethical standards of the institutional and/or national research committee(s) and with the Helsinki Declaration (as revised in 2013).

Open Access Statement: This is an Open Access article distributed in accordance with the Creative Commons Attribution-NonCommercial-NoDerivs 4.0 International License (CC BY-NC-ND 4.0), which permits the noncommercial replication and distribution of the article with the strict proviso that no changes or edits are made and the original work is properly cited (including links to both the formal publication through the relevant DOI and the license). See: https://creativecommons.org/licenses/by-nc-nd/4.0/.

\section{References}

1. Pang Z, Raudonis R, Glick BR, et al. Antibiotic resistance in Pseudomonas aeruginosa: mechanisms and alternative therapeutic strategies. Biotechnol Adv 2019;37:177-92.

2. Cornaglia G, Giamarellou H, Rossolini GM. Metallo- $\beta$ lactamases: a last frontier for $\beta$-lactams? Lancet Infect Dis 2011;11:381-93.

3. Wi YM, Choi JY, Lee JY, et al. Antimicrobial Effects of $\beta$-Lactams on Imipenem-Resistant CeftazidimeSusceptible Pseudomonas aeruginosa. Antimicrob Agents Chemother 2017;61:e00054-17.

4. Ochs MM, Bains M, Hancock RE. Role of putative loops 2 and 3 in imipenem passage through the specific porin OprD of Pseudomonas aeruginosa. Antimicrob Agents Chemother 2000;44:1983-5.

5. Riera E, Cabot G, Mulet X, et al. Pseudomonas aeruginosa carbapenem resistance mechanisms in Spain: impact on the activity of imipenem, meropenem and doripenem. J Antimicrob Chemother 2011;66:2022-7.

6. Epp SF, Köhler T, Plésiat P, et al. C-terminal region of Pseudomonas aeruginosa outer membrane porin $\mathrm{OprD}$ modulates susceptibility to meropenem. Antimicrob Agents Chemother 2001;45:1780-7.

7. Wang J, Zhou JY, Qu TT, et al. Molecular epidemiology and mechanisms of carbapenem resistance in Pseudomonas aeruginosa isolates from Chinese hospitals. Int $\mathrm{J}$ Antimicrob Agents 2010;35:486-91.

8. Okamoto K, Gotoh N, Nishino T. Extrusion of penem antibiotics by multicomponent efflux systems MexABOprM, MexCD-OprJ, and MexXY-OprM of Pseudomonas aeruginosa. Antimicrob Agents Chemother 2002;46:2696-9.

9. Chalhoub H, Sáenz Y, Nichols WW, et al. Loss of activity of ceftazidime-avibactam due to MexAB-OprM efflux and overproduction of AmpC cephalosporinase in Pseudomonas aeruginosa isolated from patients suffering from cystic fibrosis. Int J Antimicrob Agents 2018;52:697-701.

10. Sacha P, Wieczorek P, Hauschild T, et al. Metallobeta-lactamases of Pseudomonas aeruginosa--a novel mechanism resistance to beta-lactam antibiotics. Folia Histochem Cytobiol 2008;46:137-42.

11. Bellés A, Bueno J, Rojo-Bezares B, et al. Characterisation of VIM-2-producing Pseudomonas aeruginosa isolates 
from lower tract respiratory infections in a Spanish hospital. Eur J Clin Microbiol Infect Dis 2018;37:1847-56.

12. Zhou XY, Kitzis MD, Gutmann L. Role of cephalosporinase in carbapenem resistance of clinical isolates of Pseudomonas aeruginosa. Antimicrob Agents Chemother 1993;37:1387-9.

13. Drawz SM, Bonomo RA. Three decades of beta-lactamase inhibitors. Clin Microbiol Rev 2010;23:160-201.

14. Moyá B, Beceiro A, Cabot G, et al. Pan-s-lactam resistance development in Pseudomonas aeruginosa clinical strains: molecular mechanisms, penicillin-binding protein profiles, and binding affinities. Antimicrob Agents Chemother 2012;56:4771-8

15. Salih W, Schembri S, Chalmers JD. Simplification of the IDSA/ATS criteria for severe CAP using meta-analysis and observational data. Eur Respir J 2014;43:842-51.

Cite this article as: He L, He A, Qian Y. Imipenem-resistant Pseudomonas aeruginosa treated with piperacillin/tazobactam in a patient with severe pneumonia: a case report and related literature review. Ann Palliat Med 2021;10(1):810-817. doi: 10.21037/apm-20-2495
16. Singh D, Agusti A, Anzueto A, et al. Global Strategy for the Diagnosis, Management, and Prevention of Chronic Obstructive Lung Disease: the GOLD science committee report 2019. Eur Respir J 2019;53:1900164.

17. Gallego M, Pomares X, Espasa M, et al. Pseudomonas aeruginosa isolates in severe chronic obstructive pulmonary disease: characterization and risk factors. BMC Pulm Med 2014;14:103.

18. Huang X, Deng ZD, Ni YX, et al. Chinese experts' consensus on prevention and control of multidrug resistance organism healthcare-associated infection. Chinese Journal of Infection Control 2015;14:1-9.

19. Musher DM, Thorner AR. Community-acquired pneumonia. N Engl J Med 2014;371:1619-28.

(English Language Editor: A. Kassem) 